\title{
Cellular organization and histogenesis of adenosquamous carcinoma of the pancreas: evidence supporting the squamous metaplasia concept
}

\author{
Werner Boecker ${ }^{1,4,5} \cdot$ Katharina Tiemann $^{2} \cdot$ Joerg Boecker $^{3} \cdot$ Marieta Toma $^{4} \cdot$ Michael H. Muders $^{4} \cdot$ Thomas Löning $^{5}$. \\ Igor Buchwalow ${ }^{2} \cdot$ Karl J. Oldhafer $^{6}$. Ulf Neumann ${ }^{3} \cdot$ Bernd Feyerabend $^{7} \cdot$ Andre Fehr $^{8} \cdot$ Göran Stenman $^{8}$
}

Accepted: 2 March 2020 / Published online: 13 March 2020

(c) The Author(s) 2020

\begin{abstract}
Adenosquamous carcinoma of the pancreas (ASCAP) is characterized by conventional pancreatic ductal adenocarcinoma (PDAC) and squamous carcinoma components with at least $30 \%$ of the tumour showing squamous differentiation. To get further insight into the histogenesis of these lesions, we analysed the cellular organization of ASCAP compared to PDACs. Using Immunohistochemistry and triple immunofluorescence labelling studies for keratins, p63, p40, MUC1, MUC2, MUC5AC, Ki67, and EGFR we demonstrate that many ASCAPs contain a transitional zone between the K8/18-positive adenocarcinomatous component and the $\mathrm{p} 63+/ \mathrm{p} 40+/ \mathrm{K} 5 / \mathrm{K} 14+$ squamous component initiated by the expression of $\mathrm{p} 63$ in $\mathrm{K} 8 / 18+$ adenocarcinomatous cells and the appearance of basally located p63+K5/14+ cells. p63+K5/14+ cells give rise to fully developed squamous differentiation. Notably, $25 \%$ of conventional PDACs without histologically recognizable squamous component contain foci of $\mathrm{p} 63+\mathrm{p} 40+$ and $\mathrm{K} 5 / 14+$ cells similar to the transitional zone. Our data provide evidence that the squamous carcinoma components of ASCAPs originate from pre-existing PDAC via transdifferentiation of keratin K8/18-positive glandular cells to p63-, p40-, and keratin K5/14-positive squamous carcinoma cells supporting the squamous metaplasia hypothesis. Thus our findings provide new evidence about the cellular process behind squamous differentiation in ASCAPs.
\end{abstract}

Keywords Adenosquamous carcinoma $\cdot$ Pancreas $\cdot$ Keratins $\cdot$ p63 $\cdot$ p40 $\cdot$ MUC1 $\cdot$ MUC5AC $\cdot$ Ki67 $\cdot$ Transdifferentiation

\section{Abbreviations}

ASCAP Adenosquamous carcinoma of the pancreas

PDAC Pancreatic ductal adenocarcinoma
K Keratin

CEA Carcino-embryonal antigen

Werner Boecker, Katharina Tiemann, Joerg Boecker contributed equally to this work.

Electronic supplementary material The online version of this article (https://doi.org/10.1007/s00418-020-01864-y) contains supplementary material, which is available to authorized users.

Werner Boecker

boeckerwj@gmail.com

$\checkmark$ Igor Buchwalow

buchwalow@pathologie-hh.de

1 Gerhard-Domagk Institute of Pathology, University of Münster, Münster, Germany

2 Department of Pathology/Hematopathology, Institute for Hematopathology, Fangdieckstr. 75, 22547 Hamburg, Germany

3 Department of Surgery and Transplantation, University Hospital RWTH Aachen, Aachen, Germany
4 Institute of Pathology, University of Bonn, Bonn, Germany

5 Gerhard-Seifert Reference Center for Oral-, Gyneco-, and Breast Pathology, Hamburg, Germany

6 Department of Surgery, Asklepius Clinic Barmbek, Hamburg, Germany

7 Department of Pathology, Asklepius Clinic Barmbek, Hamburg, Germany

8 Sahlgrenska Cancer Center, Department of Pathology, University of Gothenburg, SE- 40530 Gothenburg, Sweden 


\section{Introduction}

Adenosquamous carcinoma of the pancreas (ASCAP) is a rare histological subtype of pancreatic ductal adenocarcinoma (PDAC) (Boyd et al. 2012; Gill et al. 2019; Imaoka et al. 2014; Katz et al. 2011; Murakami et al. 2003; Okabayashi and Hanazaki 2008; Simone et al. 2013; Voong et al. 2010). According to the WHO-classification of pancreatic carcinomas, ASCAP is characterized by variable proportions of glandular and squamous carcinoma component and the squamous component should account for at least $30 \%$ of the tumour to qualify as ASCAP (Gill et al. 2019).

Several hypotheses have been proposed regarding the histogenesis of ASCAP, including the differentiation-, the squamous metaplasia-, and the collision theory (Borazanci et al. 2015; Kardon et al. 2001; Madura et al. 1999; Motojima et al. 1992). According to the differentiation theory, the squamous and adenocarcinoma components of ASCAP develop from the same progenitor cancer cells. This theory is supported by recent molecular findings demonstrating similar genomic alterations in the two components of ASCAP (Fang et al. 2017; Marcus et al. 2017). A similar model was recently proposed for low grade adenosquamous carcinoma/syringoma of the breast, tumours which also contain squamous and glandular elements (Boecker et al. 2015, 2017). The squamous metaplasia theory suggests that the malignant squamous component of ASCAP originate from pre-existing PDAC (Kardon et al. 2001; Yamaguchi and Enjoji 1991). Finally, the collision theory, suggesting that the glandular and squamous components arise independently from each other and subsequently merge to one tumour, and the proposal that squamous metaplasia of the pancreatic ductal epithelium, occurring in the setting of chronic pancreatitis, contributes to the development of ASCAP (Simone et al. 2013), are nowadays abandoned by most authors (Kardon et al. 2001).

We hypothesize that the cellular organization of these tumours gives us further insight into the histogenesis of these lesions. We, therefore, analysed the cellular organization of 25 ASCAPs compared to 20 PDACs. We used immunohistochemistry and triple immunofluorescence stainings for p63, p40, different keratins, MUC1, MUC2, MUC5AC, the proliferation marker Ki67, and EGFR. Notably, we detected transdifferentiation of pre-existing glandular elements to squamous elements and subscribe to the theory that adenosquamous carcinomas in the pancreas occur as a result of malignant squamous metaplastic change of an adenocarcinoma.
Table 1 Demographic data, morphological findings, and outcome of 25 patients with ASCAP

\begin{tabular}{|c|c|}
\hline \multicolumn{2}{|l|}{ Features } \\
\hline Gender (m/f) & $10 / 15$ \\
\hline Age (median) & 71 (55-81 years) \\
\hline \multicolumn{2}{|l|}{ Site } \\
\hline Head & $18(72 \%)$ \\
\hline Tail & $7(28 \%)$ \\
\hline Size (median) & $3.92($ range $1.7-7.0 \mathrm{~cm})$ \\
\hline \multicolumn{2}{|l|}{ Histologic grade } \\
\hline Grade 1 & 0 \\
\hline Grade 2 & $7(28 \%)$ \\
\hline Grade 3 & $15(60 \%)$ \\
\hline Grade 4 & $3(12 \%)$ \\
\hline Mitosis per $10 \mathrm{HPF}$ & Mean 5.72 \\
\hline Squamous component (mean \%) & 74 \\
\hline \multicolumn{2}{|l|}{ TNM } \\
\hline pT1 & $1(4 \%)$ \\
\hline pT2 & - \\
\hline pT3 & $21(84 \%)$ \\
\hline pT4 & $3(12 \%)$ \\
\hline $\mathrm{pN} 1$ & $16(64 \%)$ \\
\hline M1 & $4(16 \%)$ \\
\hline L1 & $19(76 \%)$ \\
\hline V1 & $11(44 \%)$ \\
\hline No of nodes analysed/case & $17.95(\mathrm{SD} 8$, range 31$)$ \\
\hline Pn1 & $22(88 \%)$ \\
\hline $\mathrm{R} 1$ & $12(48 \%)$ \\
\hline PanIN & $13(52 \%)$ \\
\hline Overall survival (median) & 8.2 months \\
\hline
\end{tabular}

\section{Material and methods}

\section{Case selection}

Among 562 patients with PDAC (Table 1), who had undergone pancreas resection with curative intent from 2007-2018, twenty-five cases (4.4\%) were diagnosed as ASCAP with the squamous component accounting for at least $30 \%$ of the tumour (Gill et al. 2017). The samples were redundant clinical specimens that had been deidentified and unlinked from patient information. Formalin-fixed paraffin-embedded (FFPE) tissue blocks were retrieved from these cases and from 20 cases of PDAC from the files of the Departments of Pathology of the University of Aachen and of the Asklepius Clinic Hamburg Barmbek. As most of these tumours were classified according to the 7 th edition of UICC TNM classification (Sobin et al. 2009) we used this edition for all cases. The study was approved by the Committee for Research 
Table 2 Primary antibodies used in this study

\begin{tabular}{llll}
\hline Antibody & Clone & Source & Dilution \\
\hline p63 & 4A4 & Biocare medical & $1: 50$ \\
K5 & ER16014 & MEDAC & $1: 100$ \\
K5/6 & D5/16 B4 & Dako & $1: 50$ \\
K14 & LL002 & AbCam & $1: 50$ \\
K5/14 & EP160Y/LL002 & Cell marque & Ready to use \\
K7 & OV-TL12/30 & Dako & $1: 50$ \\
K18-FITC & CY-90 & Sigma & $1: 50$ \\
K8/18 & 5D3 & Zytomed & $1: 50$ \\
EMA & E 29 & Ventana & Ready to use \\
MUC1 & NCL-MUC1 & Novocastra & $1: 50$ \\
MUC2 & Ma695 & & \\
& NCL-MUC2 & Novocastra & $1: 50$ \\
MUC5AC & MRQ-19 & Cell marque & Ready to use \\
EGFR & 3C6 & Ventana & Ready to use \\
Ki67 & SP6 & Thermo fisher & $1: 100$ \\
\hline
\end{tabular}

Ethics (review board) of the University of Aachen and by the Ethic Board of the Institute of Hematopathology Hamburg.

\section{Histopathology and immunohistochemistry}

Histological grading of the ductal component was performed according to the current WHO-classification of pancreatic tumours (Gill et al. 2019). Carcinomas containing a large amount of anaplatic/sarcomatoid differentiations were classified as grade 4. FFPE tissue sections ( $4 \mu \mathrm{m}$ thick) were pre-treated and stained as described elsewhere (Buchwalow et al. 2011). Immunostains and triple immunofluorescence stainings were performed using antibodies raised against different keratins, p63, p40, CEA, EGFR, MUC1, MUC2, MUC5AC, p53, and Ki67. The sources and dilutions of all antibodies are shown in Table 2. The four different antibodies against the highmolecular weight keratins K5 and K14 (Table 2) yielded identical results, so that the term "K5/14" is used throughout the text. All markers were scored separately and quantified as percentages of the total number of tumour cells counted in 25 cases of ASCAP and 20 cases of PDAC.

\section{Results}

\section{Clinicopathological features}

The clinico-pathological characteristics of the patients with ASCAP, including 15 females and 10 males, are shown in Table 1. The median age of the patients was 71 years (range 55-81) and the median size of the tumors was $3.92 \mathrm{~cm}$ (range 1.7-7.0 cm). Eighteen tumors were located in the head and seven in the tail of the pancreas. The tumors had a mean squamous component of about $70 \%$ and were graded $2(28 \%), 3(60 \%)$, or $4(12 \%)$. The median overall survival of the patients was 8.2 months.

\section{Transitional zones occur between the adenocarcinomatous and squamous carcinoma components}

ASCAP typically demonstrated a patchwork pattern of adenocarcinoma and squamous carcinoma components of variable grades and proportions, haphazardly arranged in a mosaic pattern (Figs. 1a-c, 2a, b). The entire spectrum of glandular growth and cellular patterns seen in conventional PDACs were observed also in this series of ASCAPs. The squamous components included the features known to occur in conventional squamous carcinoma in other sites. Notably, transitional zones between the glandular and squamous components were identified in most cases (see below).

\section{p63 and K5/14 positive cells are the likely source of the squamous carcinoma components of ASCAP}

The immunohistological features of 25 ASCAP and 20 PDAC cases are summarized in Table 3. Strong nuclear p53-immunostaining was observed in $95.8 \%$ (23/24) of ASCAP cases as opposed to 60\% (12/20) of PDAC-cases. No MUC2 immunostaining was found in ASCAP- and PDACcases included in this study. Three different epithelial phenotypic patterns were observed in most tumours of our series of ASCAP cases (Figs. 1, 2, 3 and Supplementary Figs. 2, 3): (1) Areas with pure adenocarcinoma resembling pancreatobiliary-type adenocarcinomas of PDAC was characterized by the expression of K8/18, K7, MUC5AC, MUC1, and CEA. (2) Areas with squamous carcinoma which are positive for $\mathrm{p} 63$, p40, K5/14, and variably positive for K10/13. The squamous components overexpressed EGFR in $94.7 \%$ of ASCAP cases compared to $20 \%$ in PDAC. K8/18 and MUC5AC were not expressed or expressed at low levels in well differentiated squamous carcinoma but were usually expressed, albeit at lower intensity, in poorly differentiated 
Fig. 1 Histological and immunohistochemical features of adenosquamous carcinoma of the pancreas; all pictures are derived from the same tumour a Classical patchwork pattern with abrupt transition between adenocarcinomatous (hashtag) and squamous elements (asterisks); b-c higher magnification of squamous elements of the same tumour with nonkeratinizing (b) and keratinizing features (c); d-i immunostainings showing positivity for $\mathrm{K} 8 / 18$ and Muc5AC in the glandular component (hashtags in $\mathbf{f}$ and $\mathbf{h}$ ) and diffuse robust staining for p63, K5/14, and EGFR in the squamous component (asterisks in $\mathbf{d}, \mathbf{e}, \mathbf{g}$, and i). Scale bar $100 \mu \mathrm{m}$
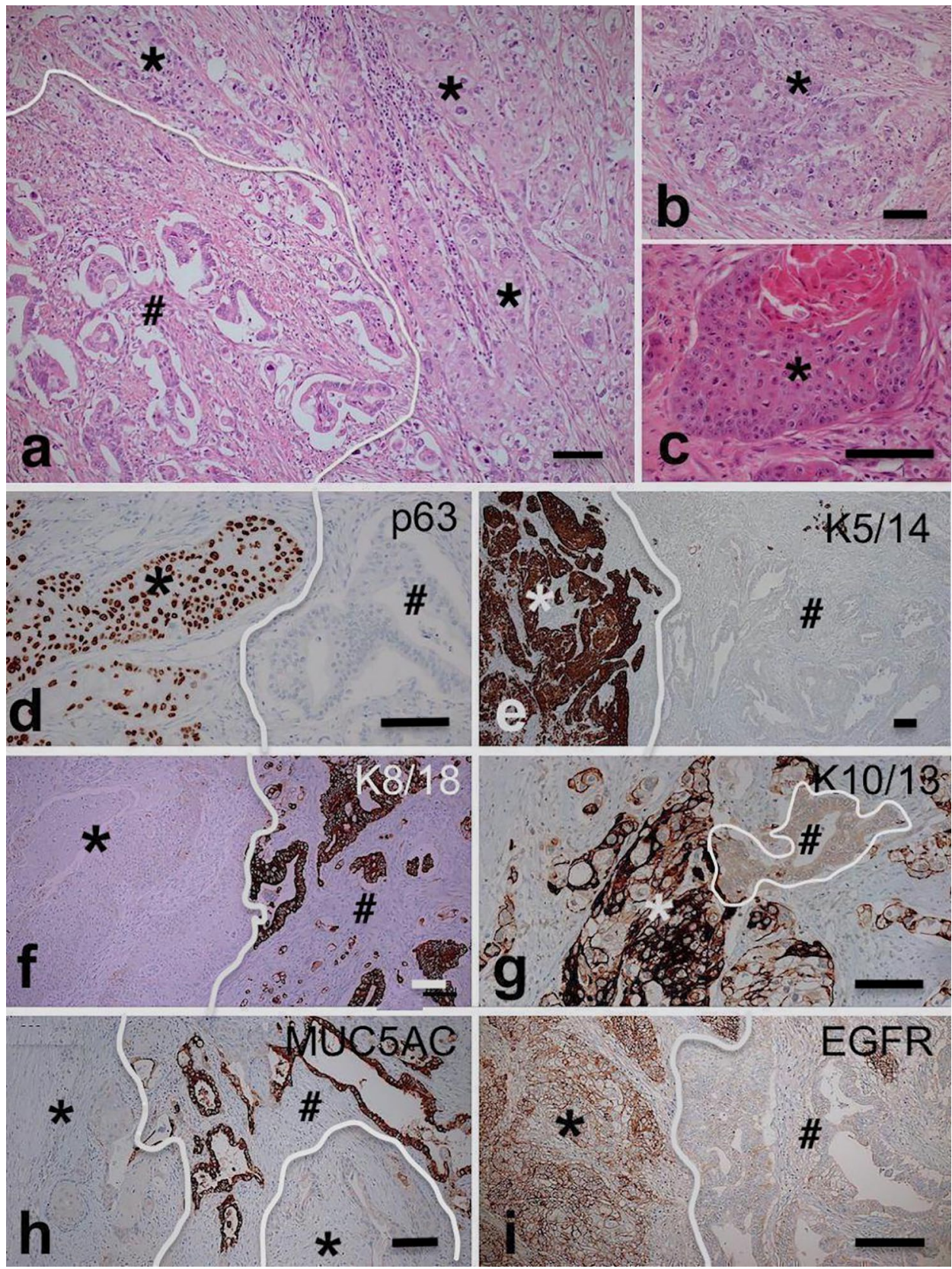

squamous carcinoma; In contrast to the K8/18 staining, decreased positive $\mathrm{K} 7$-staining was usually observed in the squamous component. (3) Areas of transitional zones were detected in 16 of the 25 ASCAP cases which were preferentionally localized at the interface between the adenocarcinomatous and the squamous carcinoma areas. These transitional zones are characterized by $\mathrm{K} 8 / 18+$ glandular components that coexpress p63 and, furthermore, show p63+, K5/14+ cells in basal position (Figs. 2c-f, 3c-e and Supplementary Fig. 1b-d). However, similar phenomena were also found in adenocarcinomatous areas which histologically did not disclose any squamous differentiation. The proliferation of $\mathrm{p} 63+/ \mathrm{K} 5 / 14+$ cells leads to the formation of squamous structures (Figs. 1, 2, 3 and Supplementary Figs. 1, 2). Importantly, most of the p63 + cells also stain for $\mathrm{p} 40$ (Figs. $2 \mathrm{~h}-\mathrm{i}, 3 \mathrm{e}-\mathrm{e}$ "). Taken together, we hypothesize that the p63+K5/14+ cells are the source of the squamous carcinoma components.

Pancreatic Intraepithelial Neoplasia 3 (PanIN 3) was observed in $52 \%$ of the ASCAP cases. In one of these, a focal positivity for $\mathrm{p} 63+$ and $\mathrm{K} 5 / 14+$ of a PanIN lesion was found in pancreatic tissue not involved by invasive cancer (Supplementary Fig. 3). 
Fig. 2 Immunhistological hallmark of the transitional zone in regard to p63- and p40-staining; a-b typical patchwork pattern of adenosquamous carcinoma in HE-staining, illustrated by $\mathrm{p} 40$-negative staining in the glandular component (b left) and p40-positivity in the squamous component (b right, see also $\mathbf{h}$ and $\mathbf{i})$; $\mathbf{c}-\mathbf{f}$ transitional zone with increasing expression of p63 in glandular structures $(\mathbf{d}, \mathbf{e}, \mathbf{f})$ characterized by the appearance of single or groups of p63 + cells; these cells are partly luminally and partly basally located (compare Fig. 3); g-i squamous component which shows robustly p63(h) and p40 staining (i), inset in (g) shows k10/13-positivity in the squamous component. Adeno adenocarcinoma; Squam squamous carcinoma; trans transitional zone. Scale bar $100 \mu \mathrm{m}$
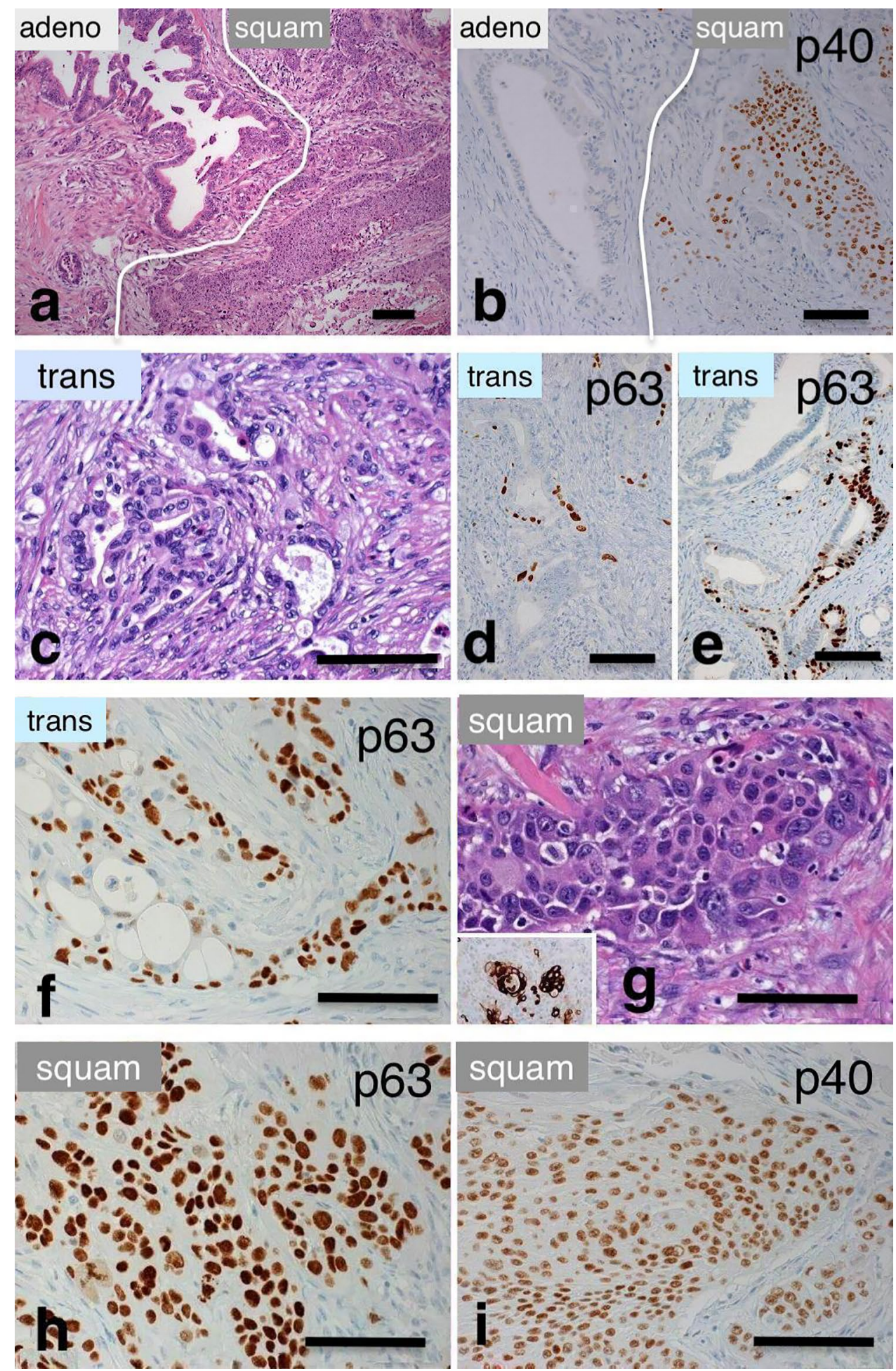

\section{PDAC and associated PanIN 3 lesions contain foci of p63+ and k5/14+ tumour cells similar to the transitional zone in ASCAP}

Twenty-five percent of conventional PDACs without any histological evidence of squamous differentiation contain foci of p63+ and K5/14+ cells (Supplementary Fig. 5) with a median of $15 \%$ of p63-immunopositive tumour cells (range 10-25\%). Associated PanIN 3 lesions were found in 10 cases (50\%) of PDAC. In one of these lesions p63+, K5/14+ cells were found which even expressed the squamous keratins 


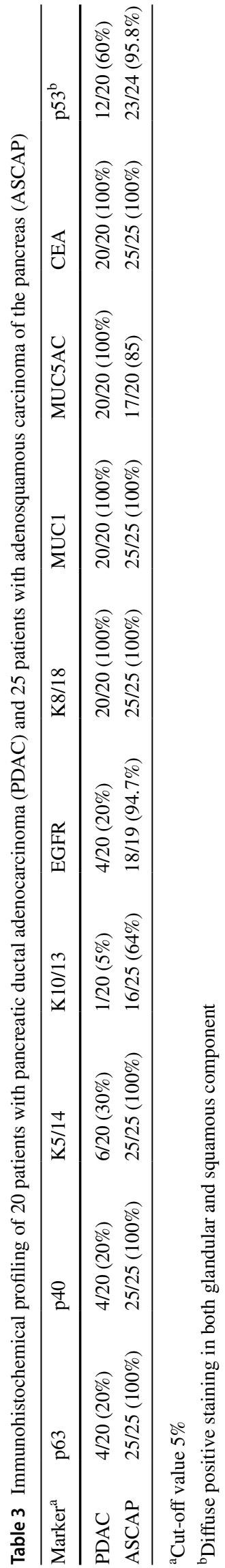

K10/13 and EGFR in a histologically classical PanIN 3 lesion.

\section{Discussion}

Here, we aim to get further insight into the histogenesis of ASCAP by analysing the cellular composition and differentiation of these lesions using immunohistochemistry and triple immunofluorescence stainings. The adenocarcinomatous component shows a pronounced morphological and immunophenotypic similarity to classical PDAC with positivity for K8/18, MUC1, and MUC5AC. Notably, the squamous lineage of these lesions is identical to squamous carcinomas in other anatomical locations and its hallmark is the robust positive staining for p63 (Uramoto et al. 2010), p40, and $\mathrm{K} 5 / 14$ in all cases, with coexpression of the EGFR in $94.7 \%$, and variable staining for the squamous keratins K10/13.

Based on the data of our study, we present new evidence in support of a transdifferentiation of adenocarcinoma to squamous carcinoma in ASCAP consistent with the squamous metaplasia theory (Cihak et al. 1972) (Fig. 4). This hypothesis is supported by the presence of clearly recognizable transitional zones between the K8/18 + MUC5AC + adenocarcinomatous and the p $63+\mathrm{K} 5 / 14+$ squamous components. The transitional zone is characterized by the early appearance of nuclear p63 expression in $\mathrm{K} 8 / 18+$ glandular cells and the occurrence of p $63+\mathrm{K} 5 / 14+$ basally located cells. These cells subsequently proliferate and differentiate forming the squamous components of ASCAP. Notably, in all cases most of the p63 + cells stained robustly for $\mathrm{p} 40$ (Fig. 3) and in 64\% also for the squamous keratins K10/13. Thus, it seems as if the K8/18 + glandular cells of ASCAP transform to $\mathrm{p} 63+/ \mathrm{p} 40+/ \mathrm{K} 5 / 14+$ cells which, therefore, constitute the squamous metaplastic epithelial proliferations in these tumours. The findings indicate that p63 and p40 are key players in this metaplastic process. This is in line with reports showing that deltaNp63, i.e. p40, is the most widely expressed isoform in squamous carcinoma, compatible with a role for this protein in promoting the growth of neoplastic cell in these tissues (Nylander et al. 2002; Reis-Filho et al. 2002).

Our interpretation finds its analogy in the embryogenetic development of several epithelia (Daniely et al. 2004; Di Como et al. 2002; Koster et al. 2004; Koster and Roop 2004b; Signoretti et al. 2000). Early embryonic mouse ectoderm shows transformation of single layered K8/18+ cells to multi-layered squamous epithelium with p63-positivity in basal keratinocytes (Koster and Roop 2004a). In this context p63 appears to initiate epithelial stratification and to maintain proliferative potential of basal keratinocytes in mature squamous epithelium (Senoo et al. 2007). Similarly, the tracheobronchial epithelium in mice consists of a layer of two 
Fig. 3 Triple immunostainings demonstrate the process of squamous differentiation in tumour glands; $\mathbf{a}-\mathbf{b}$ a tumour gland showing focal squamous differentiation (asterisks) characterized by coexpression of p63 and K5/14; c-d these pictures demonstrate earlier developments with the expression of p63-positivity in K8/18 + glands (arrows) and basally located p63+K5/14 + cells (asterisks in c-d); e-e"' Notice that most p63 + cells robustly co-express p40 (arrows). Occasional cells express p63 but lack p40 (broken arrows). Scale bar $100 \mu \mathrm{m}$

Fig. 4 Hypothetical model of adenosquamous carcinoma of the pancreas a: triple immunofluorescence demonstrating the expression of $\mathrm{p} 63$ in the $\mathrm{k} 8 / 18$ + glandular cells (small arrows) and the basally located p63 + K5/14 + cells (large arrows) (this figure contains the same epithelial structure as in Fig. 3c); b hypothetical cellular model explaining the development of the glandular and squamous components in these tumours. Scale bar $100 \mu \mathrm{m}$
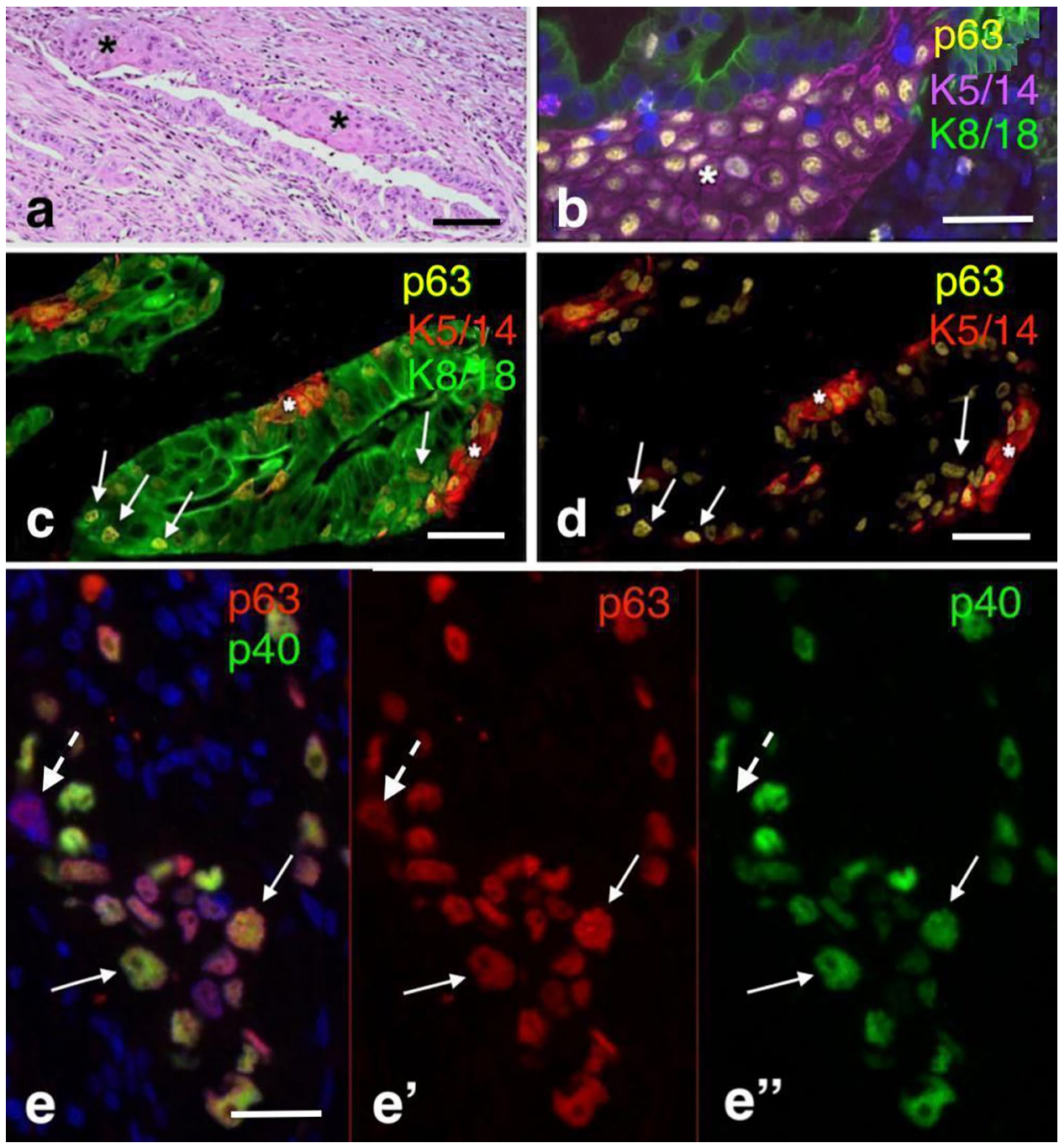

\section{Adenosquamous Carcinoma of the Pancreas}

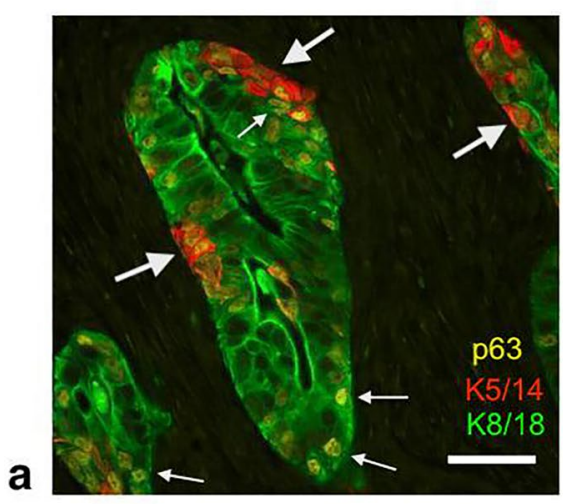

Squamous Carcinoma

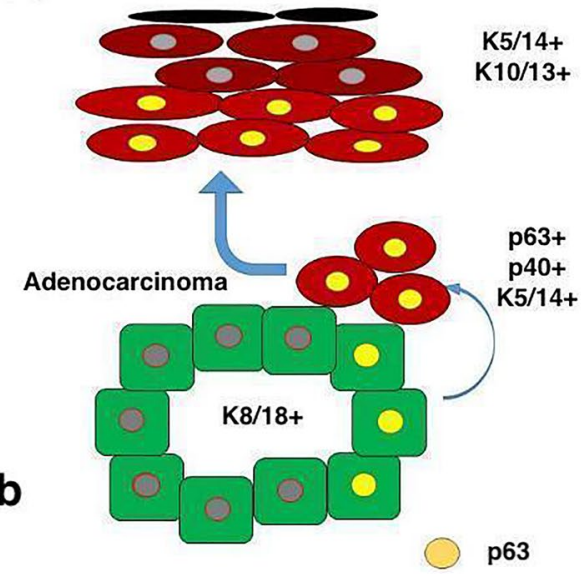


to three cells. During development, stem cells first differentiate into p63-negative ciliated and secretory cells and later, at birth, they begin to differentiate into $\mathrm{p} 63+\mathrm{K} 14+$ basal cells (Daniely et al. 2004).

Additional evidence in favour of the squamous metaplasia theory for ASCAP comes from our finding that one fourth of unselected cases of the PDACs without histologically recognizable squamous differentiation contained small areas with p63+, and K5/14 + cells and even p40+ and K10/13+ cell differentiations. Expression of squamous type keratins in PDAC and their possible relation to squamous metaplasia have already been discussed as early as 1989 (Moll et al. 1989; Schussler et al. 1992). Collectively, we, therefore, suggest that these findings are indicative of an abortive squamous metaplasia in otherwise classical PDACs and we suggest that these findings might express the metaplastic potential of the neoplastic ductal epithelium.

Furthermore, 54\% of our ASCAP-cases were associated with PanIN 3 changes. As most invasive PDACs are thought to develop through the adenoma-carcinoma pathway and, based on the assumption that the squamous component originates from the adenocarcinoma, it is reasonable to postulate a developmental sequence from PanIN via PDAC to ASCAP. In line with this argument are case reports that have described the association of ASCAP with intraductal papillary mucinous neoplasia and suggested a model of adenomacarcinoma progression for these tumours (Martinez de Juan et al. 2017; Matsuzaka et al. 2016).

Finally, indirect evidence in favour of our model comes from previous studies showing that several glandular epithelia which contain p $63+\mathrm{K} 5 / 14$ + basal cells do not express p63 in the differentiated glandular compartment (Boecker et al. 2019; 2014; Daniely et al. 2004; Di Como et al. 2002; Nylander et al. 2002; Signoretti et al. 2000). Furthermore, in contrast to the findings in this ASCAP study, low-grade adenosquamous carcinoma/syringomatous tumours of the breast, which contain p63 + and K5/14 + cells, first downregulate p63 and subsequently generate the $\mathrm{K} 8 / 18$ + glandularly differentiated cells via p63-negative $\mathrm{K} 5 / 14$ + intermediary cells indicating a different histogenetic pathway in these lesions compared to ASCAP tumours (Boecker et al. 2019; 2014).

In conclusion, based on the findings of the cellular organization of ASCAP in this study, we subscribe to the theory that these tumours occur as a result of malignant squamous metaplastic change of an adenocarcinoma. Our findings suggest that the squamous carcinoma component originates from a pre-existing K8/18 + PDAC through transdifferentiation of glandular cells to p63+, p40+, and $\mathrm{K} 5 / 14+$ squamous carcinoma cells. Additional studies will be needed to fully understand the molecular mechanisms behind these interesting cellular differentiation processes.
Acknowledgements We thank our German colleagues for sharing the reagents and tissue samples as well as sending us the material as consultation cases. Part of this study was supported by a grant from the Swedish Cancer Society and BioCARE—a National Strategic Research Program at University of Gothenburg.

Author contributions WB, KT and JB conceived the study and wrote the manuscript. MT, MHM, ThL, KJO, UN, BF, AF and GS selected and analysed the cases and participated in the design of the study. IB, WB did the immunohistochemistry. All the authors reviewed and edited the manuscript.

\section{Compliance with ethical standards}

Conflict of interest The authors declare no competing financial interests.

Open Access This article is licensed under a Creative Commons Attribution 4.0 International License, which permits use, sharing, adaptation, distribution and reproduction in any medium or format, as long as you give appropriate credit to the original author(s) and the source, provide a link to the Creative Commons licence, and indicate if changes were made. The images or other third party material in this article are included in the article's Creative Commons licence, unless indicated otherwise in a credit line to the material. If material is not included in the article's Creative Commons licence and your intended use is not permitted by statutory regulation or exceeds the permitted use, you will need to obtain permission directly from the copyright holder. To view a copy of this licence, visit http://creativecommons.org/licenses/by/4.0/.

\section{References}

Boecker W, Reusch M, Mielke V, Reusch U, Loening T, Tiemann M, Buchwalow I (2019) Spatial analysis of p63, K5 and K7 defines two groups of progenitor cells that differentially contribute to the maintenance of normal sebaceous glands, extraocular sebaceous carcinoma and benign sebaceous tumors. J Dermatol 46:249-258

Boecker W, Stenman G, Loening T, Andersson MK, Berg T, Lange A, Bankfalvi A, Samoilova V, Tiemann K, Buchwalow I (2015) Squamous/epidermoid differentiation in normal breast and salivary gland tissues and their corresponding tumors originate from p63/K5/14-positive progenitor cells. Virchows Arch 466:21-36

Boecker W, Stenman G, Loening T, Andersson MK, Sinn HP, Barth P, Oberhellmann F, Bos I, Berg T, Marusic Z, Samoilova V, Buchwalow I (2014) Differentiation and histogenesis of syringomatous tumour of the nipple and low-grade adenosquamous carcinoma: evidence for a common origin. Histopathology 65:9-23

Boecker W, Stenman G, Schroeder T, Schumacher U, Loening T, Stahnke L, Lohnert C, Siering RM, Kuper A, Samoilova V, Tiemann M, Korsching E, Buchwalow I (2017) Multicolor immunofluorescence reveals that $\mathrm{p} 63$ - and/or K5-positive progenitor cells contribute to normal breast epithelium and usual ductal hyperplasia but not to low-grade intraepithelial neoplasia of the breast. Virchows Arch 470:493-504

Borazanci E, Millis SZ, Korn R, Han H, Whatcott CJ, Gatalica Z, Barrett MT, Cridebring D, Von Hoff DD (2015) Adenosquamous carcinoma of the pancreas: molecular characterization of 23 patients along with a literature review. World J Gastrointest Oncol 7:132-140

Boyd CA, Benarroch-Gampel J, Sheffield KM, Cooksley CD, Riall TS (2012) 415 patients with adenosquamous carcinoma of the 
pancreas: a population-based analysis of prognosis and survival. J Surg Res 174:12-19

Buchwalow I, Samoilova V, Boecker W, Tiemann M (2011) Non-specific binding of antibodies in immunohistochemistry: fallacies and facts. Sci Rep 1:28

Cihak RW, Kawashima T, Steer A (1972) Adenoacanthoma (adenosquamous carcinoma) of the pancreas. Cancer 29:1133-1140

Daniely Y, Liao G, Dixon D, Linnoila RI, Lori A, Randell SH, Oren M, Jetten AM (2004) Critical role of p63 in the development of a normal esophageal and tracheobronchial epithelium. Am J Physiol Cell Physiol 287:C171-181

Di Como CJ, Urist MJ, Babayan I, Drobnjak M, Hedvat CV, TeruyaFeldstein J, Pohar K, Hoos A, Cordon-Cardo C (2002) p63 expression profiles in human normal and tumor tissues. Clin Cancer Res 8:494-501

Fang Y, Su Z, Xie J, Xue R, Ma Q, Li Y, Zhao Y, Song Z, Lu X, Li H, Peng C, Bai F, Shen B (2017) Genomic signatures of pancreatic adenosquamous carcinoma (PASC). J Pathol 243:155-159

Gill AJ, Klimstra DJ, Lam AK, Washington MK (2019) Tumours of the Pancreas. In: Arends MJ, Fukayama M, Klimstra DS, et.al (eds) Digestive System Tumours, vol. 1. WHO classification of tumours series, 5th edition, Lyon (France), pp 296-372

Gill I, Brezina L, Siddiqi J (2017) Unexpected case of sclerosing mucoepidermoid carcinoma of the submandibular salivary gland. Br J Oral Maxillofac Surg 55:646-647

Imaoka H, Shimizu Y, Mizuno N, Hara K, Hijioka S, Tajika M, Kondo S, Tanaka T, Ogura T, Obayashi T, Hasegawa T, Niwa Y, Yamao $\mathrm{K}$ (2014) Clinical characteristics of adenosquamous carcinoma of the pancreas: a matched case-control study. Pancreas 43:287-290

Kardon DE, Thompson LD, Przygodzki RM, Heffess CS (2001) Adenosquamous carcinoma of the pancreas: a clinicopathologic series of 25 cases. Mod Pathol 14:443-451

Katz MH, Taylor TH, Al-Refaie WB, Hanna MH, Imagawa DK, AntonCulver H, Zell JA (2011) Adenosquamous versus adenocarcinoma of the pancreas: a population-based outcomes analysis. J Gastrointest Surg 15:165-174

Koster MI, Kim S, Mills AA, DeMayo FJ, Roop DR (2004) p63 is the molecular switch for initiation of an epithelial stratification program. Genes Dev 18:126-131

Koster MI, Roop DR (2004a) The role of p63 in development and differentiation of the epidermis. J Dermatol Sci 34:3-9

Koster MI, Roop DR (2004b) Transgenic mouse models provide new insights into the role of p63 in epidermal development. Cell Cycle 3:411-413

Madura JA, Jarman BT, Doherty MG, Yum MN, Howard TJ (1999) Adenosquamous carcinoma of the pancreas. Arch Surg 134:599-603

Marcus R, Maitra A, Roszik J (2017) Recent advances in genomic profiling of adenosquamous carcinoma of the pancreas. J Pathol 243:271-272

Martinez de Juan F, Reolid Escribano M, Martinez Lapiedra C, Maia de Alcantara F, Caballero Soto M, Calatrava Fons A, Machado I (2017) Pancreatic adenosquamous carcinoma and intraductal papillary mucinous neoplasm in a CDKN2A germline mutation carrier. World J Gastrointest Oncol 9:390-396

Matsuzaka S, Karasaki H, Ono Y, Ogata M, Oikawa K, Tamakawa S, Chiba S, Muraki M, Yokochi T, Funakoshi H, Kono T, Nagashima
K, Mizukami Y (2016) Tracking the clonal evolution of adenosquamous carcinoma, a rare variant of intraductal papillary mucinous neoplasm of the pancreas. Pancreas 45:915-918

Moll R, Dhouailly D, Sun TT (1989) Expression of keratin 5 as a distinctive feature of epithelial and biphasic mesotheliomas. An immunohistochemical study using monoclonal antibody AE14. Virchows Arch B Cell Pathol Incl Mol Pathol 58:129-145

Motojima K, Tomioka T, Kohara N, Tsunoda T, Kanematsu T (1992) Immunohistochemical characteristics of adenosquamous carcinoma of the pancreas. J Surg Oncol 49:58-62

Murakami Y, Yokoyama T, Yokoyama Y, Kanehiro T, Uemura K, Sasaki M, Morifuji M, Sueda T (2003) Adenosquamous carcinoma of the pancreas: preoperative diagnosis and molecular alterations. J Gastroenterol 38:1171-1175

Nylander K, Vojtesek B, Nenutil R, Lindgren B, Roos G, Zhanxiang W, Sjostrom B, Dahlqvist A, Coates PJ (2002) Differential expression of p63 isoforms in normal tissues and neoplastic cells. J Pathol 198:417-427

Okabayashi T, Hanazaki K (2008) Surgical outcome of adenosquamous carcinoma of the pancreas. World J Gastroenterol 14:6765-6770

Reis-Filho JS, Torio B, Albergaria A, Schmitt FC (2002) p63 expression in normal skin and usual cutaneous carcinomas. J Cutan Pathol 29:517-523

Schussler MH, Skoudy A, Ramaekers F, Real FX (1992) Intermediate filaments as differentiation markers of normal pancreas and pancreas cancer. Am J Pathol 140:559-568

Senoo M, Pinto F, Crum CP, McKeon F (2007) p63 is essential for the proliferative potential of stem cells in stratified epithelia. Cell 129:523-536

Signoretti S, Waltregny D, Dilks J, Isaac B, Lin D, Garraway L, Yang A, Montironi R, McKeon F, Loda M (2000) p63 is a prostate basal cell marker and is required for prostate development. Am J Pathol 157:1769-1775

Simone CG, Zuluaga Toro T, Chan E, Feely MM, Trevino JG, George TJ Jr (2013) Characteristics and outcomes of adenosquamous carcinoma of the pancreas. Gastrointest Cancer Res 6:75-79

Sobin LH, Gospodarowitz MK, Wittekind C (2009) UICC: TNM classiofication of of malignant tumours. Wiley-Blackwell, Oxford

Uramoto H, Yamada S, Hanagiri T (2010) Immunohistochemical staining with deltaNp63 is useful for distinguishing the squamous cell component of adenosquamous cell carcinoma of the lung. Anticancer Res 30:4717-4720

Voong KR, Davison J, Pawlik TM, Uy MO, Hsu CC, Winter J, Hruban RH, Laheru D, Rudra S, Swartz MJ, Nathan H, Edil BH, Schulick R, Cameron JL, Wolfgang CL, Herman JM (2010) Resected pancreatic adenosquamous carcinoma: clinicopathologic review and evaluation of adjuvant chemotherapy and radiation in 38 patients. Hum Pathol 41:113-122

Yamaguchi K, Enjoji M (1991) Adenosquamous carcinoma of the pancreas: a clinicopathologic study. J Surg Oncol 47:109-116

Publisher's Note Springer Nature remains neutral with regard to jurisdictional claims in published maps and institutional affiliations. 